\title{
Protein Structure Prediction from NMR Hydrogen-Deuterium Exchange Data
}

Daniel R. Marzolf, ${ }^{1}$ Justin T. Seffernick, ${ }^{1}$ and Steffen Lindert ${ }^{1, *}$

${ }^{1}$ Department of Chemistry and Biochemistry, Ohio State University, Columbus, OH, 43210

* Correspondence to:

Department of Chemistry and Biochemistry, Ohio State University

2114 Newman \& Wolfrom Laboratory, 100 W. $18^{\text {th }}$ Avenue, Columbus, OH 43210

614-292-8284 (office), 614-292-1685 (fax)

lindert.1@osu.edu

\section{Individual HDX Parameter-Based Scoring Improved Model Selection Accuracy}

The SAP factor was applied to the relative solvent accessible surface area (RelSASA) and hydrogen-bonding energy (H-Bond) based scoring methods, while not included in neighbor count (NC) or order score (OS) based scoring. When using a SAP factor scaled for both parameters (scaled by 0.2 for RelSASA, and 3.0 for H-Bond), the average RMSD of the selected models improved when HDX terms were included in scoring by $0.73 \AA$ for NC, $0.93 \AA$ for RelSASA, 0.22 $\AA$ for OS, and $0.94 \AA$ for H-Bond. However, this comprised all proteins from the dataset, including those for which the selected model RMSD did not change. Of the proteins for which the selected model RMSD did not change by greater than $0.5 \AA$ ( 28 for NC, 29 for H-Bond and RelSASA, 34 for OS), the RMSD of the predicted models was below $5 \AA$ for 19 proteins for NC and 18 proteins for H-Bond, RelSASA, and OS. As such, the average selected model RMSD improvement was also calculated using only proteins for which the selected model RMSD changed by greater than $0.5 \AA$; the smallest improvement was observed in the RelSASA-based score, which resulted in an average improvement of $1.61 \AA$, however H-Bond-based scoring resulted in a greater average improvement of $4.00 \AA$.

\section{Scoring Based upon Pairs of Parameters Representing Exposure and Flexibility Improved Model Selection more than Individual Parameters Alone}

To examine the extent to which flexibility or exposure parameters contributed to scoring improvement, the individual parameters were combined in pairs that corresponded to the structural feature they represented: NC and RelSASA for exposure, OS and H-Bond for flexibility. The scores derived from pairs of parameters were then added to the Rosetta Score. Results for both flexibility- and exposure-based scoring methods are shown in Table S2. For exposure-based scoring, the scores generated from the RelSASA and NC scoring methods were added in a weighted sum, with SAP included for RelSASA-based scoring, but excluded for NC-based scoring. Using these terms to score structures, the average selected model RMSD improved by 
$0.97 \AA$, a slight increase from either of the parameters being used individually. The average RMSD for proteins in which the selected model changed by greater than $0.5 \AA$ when HDX data was included improved by $3.09 \AA$, an increase of $0.19 \AA$ from using NC or $1.48 \AA$ using RelSASA, respectively (Table S2). In flexibility-based scoring, the scores generated from the OS and H-Bond scoring methods were added as a weighted sum, with SAP included for H-Bond-based scoring but excluded for OS-based scoring. Using this, the average RMSD of selected models improved by $0.90 \AA$. However, the average RMSD for proteins in which the selected model changed by greater than $0.5 \AA$ improved by $4.36 \AA$, an increase of $0.36 \AA$ or $2.44 \AA$ compared to using H-Bond or OS individually, respectively. Figure S1 graphically depicts the RMSD of the selected model for each protein when using the Rosetta Score with and without HDX data.

Table S1. Summary of benchmark protein characteristics and selected model RMSDs before and after scoring with the combined-term HDX score.

\begin{tabular}{|c|c|c|c|c|c|c|}
\hline $\begin{array}{l}\text { PDB } \\
\text { ID }\end{array}$ & Protein Name & Organism & $\begin{array}{l}\text { Sequence } \\
\text { Length }\end{array}$ & $\begin{array}{c}\text { Number of } \\
\text { Residues with } \\
\text { Strong } \\
\text { Categorization }\end{array}$ & $\begin{array}{c}\text { Selected } \\
\text { Model RMSD } \\
\text { using Rosetta } \\
\text { Score }(\AA)\end{array}$ & $\begin{array}{c}\text { Selected } \\
\text { Model RMSD } \\
\text { using HDX } \\
\text { Score }(\AA)\end{array}$ \\
\hline 1BDD & $\begin{array}{c}\text { Immunoglobulin G- } \\
\text { binding Protein A }\end{array}$ & $\begin{array}{c}\text { Staphylococcus } \\
\text { aureus }\end{array}$ & 60 & 5 & 4.85 & 5.00 \\
\hline 1BE9 & $\begin{array}{l}\text { Disks Large } \\
\text { Homolog } 4\end{array}$ & Rattus norvegicus & 119 & 17 & 8.60 & 9.98 \\
\hline 1BTA & Barstar & $\begin{array}{c}\text { Bacillus } \\
\text { amyloliquefaciens }\end{array}$ & 89 & 6 & 1.39 & 1.74 \\
\hline $1 \mathrm{COE}$ & Cobrotoxin & Naja atra & 62 & 17 & 9.28 & 2.67 \\
\hline $1 \mathrm{HCE}$ & Histactophilin-1 & $\begin{array}{l}\text { Dicytostelium } \\
\text { discoideum }\end{array}$ & 118 & 12 & 11.62 & 2.22 \\
\hline 1HEL & Lysozyme C & Gallus gallus & 129 & 9 & 8.48 & 3.74 \\
\hline 1HFX & Alpha-lactalbumin & Cavia procellus & 123 & 7 & 3.19 & 3.19 \\
\hline 1HFZ & Alpha-lactalbumin & Bos taurus & 124 & 18 & 3.86 & 6.64 \\
\hline $1 \mathrm{JBH}$ & $\begin{array}{l}\text { Retinol-binding } \\
\text { Protein } 1\end{array}$ & Rattus norvegicus & 135 & 42 & 2.84 & 2.80 \\
\hline $1 \mathrm{JOO}$ & Thermonuclease & $\begin{array}{c}\text { Staphylococcus } \\
\text { aureus }\end{array}$ & 149 & 6 & 15.03 & 15.03 \\
\hline $1 \mathrm{MBC}$ & Myoglobin & Physeter catodon & 153 & 8 & 2.63 & 2.53 \\
\hline $1 \mathrm{MZK}$ & $\begin{array}{c}\text { Protein phosphatase } \\
\text { 2C } 70\end{array}$ & $\begin{array}{c}\text { Arabidopsis } \\
\text { thaliana }\end{array}$ & 139 & 25 & 16.00 & 13.06 \\
\hline $1 \mathrm{OK} 0$ & $\begin{array}{c}\text { Alpha-amylase } \\
\text { inhibitor HOE- } \\
467 \mathrm{~A}\end{array}$ & $\begin{array}{l}\text { Streptomyces } \\
\text { tendae }\end{array}$ & 74 & 12 & 8.67 & 4.07 \\
\hline 1OMU & Ovomucoid & $\begin{array}{l}\text { Meleagris } \\
\text { gallopavo }\end{array}$ & 56 & 14 & 3.31 & 1.67 \\
\hline $1 \mathrm{ONC}$ & Protein P-30 & Rana pipiens & 104 & 41 & 1.33 & 1.18 \\
\hline 1PGA & $\begin{array}{c}\text { Immunoglobulin G- } \\
\text { binding Protein G }\end{array}$ & $\begin{array}{c}\text { Streptococcus sp. } \\
\text { GX7805 }\end{array}$ & 56 & 9 & 0.69 & 0.70 \\
\hline $1 \mathrm{RBX}$ & $\begin{array}{l}\text { Ribonuclease } \\
\text { Pancreatic }\end{array}$ & Bos taurus & 124 & 11 & 8.74 & 4.36 \\
\hline 1RG8 & $\begin{array}{c}\text { Fibroblast Growth } \\
\text { Factor } 1 \\
\end{array}$ & Homo sapiens & 146 & 8 & 4.74 & 4.74 \\
\hline
\end{tabular}




\begin{tabular}{|c|c|c|c|c|c|c|}
\hline 1SR2 & $\begin{array}{c}\text { Phosphotransferase } \\
\text { RcsD }\end{array}$ & Escherichia coli & 116 & 53 & 18.41 & 15.02 \\
\hline 1UBQ & Ubiquitin & Homo sapiens & 76 & 13 & 3.035 & 2.58 \\
\hline $1 \mathrm{YGW}$ & $\begin{array}{l}\text { Guanyl-specific } \\
\text { ribonuclease T1 }\end{array}$ & $\begin{array}{l}\text { Aspergillus } \\
\text { oryzae }\end{array}$ & 104 & 17 & 2.307 & 4.05 \\
\hline $1 \mathrm{YMB}$ & Myoglobin & Equss caballus & 153 & 42 & 2.831 & 2.96 \\
\hline $1 \mathrm{YOB}$ & Flavodoxin-2 & $\begin{array}{l}\text { Azotobacter } \\
\text { vinelandii }\end{array}$ & 179 & 27 & 14.73 & 6.89 \\
\hline $1 Y Y X$ & $\begin{array}{c}\text { Soluble } \\
\text { Apocytochrome } \\
\text { B562 }\end{array}$ & $\begin{array}{l}\text { N/A (De Novo } \\
\text { Protein) }\end{array}$ & 106 & 19 & 3.24 & 3.41 \\
\hline $2 \mathrm{ABD}$ & $\begin{array}{l}\text { Acyl-CoA-binding } \\
\text { Protein }\end{array}$ & Bos taurus & 86 & 28 & 2.16 & 2.48 \\
\hline 2CRT & Cytotoxin 3 & Naja atra & 60 & 6 & 9.28 & 9.28 \\
\hline $2 \mathrm{EQL}$ & Lysozyme C & Equus caballus & 129 & 15 & 14.99 & 5.41 \\
\hline 2LZM & Lysozyme & $\begin{array}{l}\text { Escherichia virus } \\
\text { T4 }\end{array}$ & 164 & 27 & 8.66 & 8.66 \\
\hline 2PTL & Protein LG & $\begin{array}{c}\text { Finegoldia } \\
\text { magna ATCC } \\
29328\end{array}$ & 78 & 14 & 14.48 & 14.27 \\
\hline $2 \mathrm{VIL}$ & Villin-1 & Gallus gallus & 126 & 24 & 5.25 & 5.25 \\
\hline $3 \mathrm{~B} 0 \mathrm{O}$ & Alpha-lactalbumin & Homo sapiens & 123 & 9 & 4.44 & 4.44 \\
\hline 3BLG & Beta-lactoglobulin & Bos taurus & 162 & 24 & 14.93 & 3.77 \\
\hline $3 \mathrm{CHY}$ & $\begin{array}{c}\text { Chemotaxis Protein } \\
\text { CheY }\end{array}$ & Escherichia coli & 128 & 10 & 1.322 & 1.322 \\
\hline $3 \mathrm{CI} 2$ & $\begin{array}{c}\text { Subtilisin- } \\
\text { chymotrypsin } \\
\text { inhibitor-2A }\end{array}$ & Hordeum vulgare & 66 & 9 & 2.139 & 2.14 \\
\hline 3NP0 & $\begin{array}{l}\text { Beta-lactoglobulin, } \\
\text { unliganded form }\end{array}$ & Bos taurus & 162 & 24 & 3.76 & 2.58 \\
\hline $451 \mathrm{C}$ & Cytochrome C-551 & $\begin{array}{l}\text { Pseudomonas } \\
\text { aeruginosa }\end{array}$ & 82 & 13 & 6.01 & 6.01 \\
\hline 5PTI & $\begin{array}{c}\text { Pancreatic Trypsin } \\
\text { Inhibitor }\end{array}$ & Bos taurus & 58 & 7 & 1.316 & 1.535 \\
\hline 9PCY & Plastocyanin & $\begin{array}{l}\text { Phaseolus } \\
\text { vulgaris }\end{array}$ & 99 & 28 & 5.228 & 5.228 \\
\hline
\end{tabular}


Table S2. Summary of results following scoring for exposure- and flexibility-based HDX scoring. Selected model RMSD improvement and increase are defined by a change in selected model RMSD by greater than $-0.5 \AA$ and $0.5 \AA$, respectively.

\begin{tabular}{|c|c|c|c|c|}
\cline { 2 - 5 } \multicolumn{1}{c|}{} & $\begin{array}{c}\text { Average } \mathbf{\Delta R M S D} \\
\text { of All Proteins }(\AA)\end{array}$ & $\begin{array}{c}\text { Average } \mathbf{\Delta R M S D} \text { of Proteins } \\
\text { with Changes in RMSD }(\AA)\end{array}$ & $\begin{array}{c}\text { Number of Proteins with } \\
\text { RMSD Improved }\end{array}$ & $\begin{array}{c}\text { Number of } \\
\text { Proteins with } \\
\text { RMSD Increased }\end{array}$ \\
\hline Exposure & -0.97 & -3.09 & 8 & 3 \\
\hline Flexibility & -0.90 & -4.36 & 8 & 0 \\
\hline
\end{tabular}

Figure S1. The selected model RMSD for the Rosetta prediction and when scoring with the exposure-based (top) and flexibility-based (bottom) terms. Markers below the $\mathrm{y}=\mathrm{x}$ line indicate a protein with an improvement in selected model RMSD, with those above worsening.

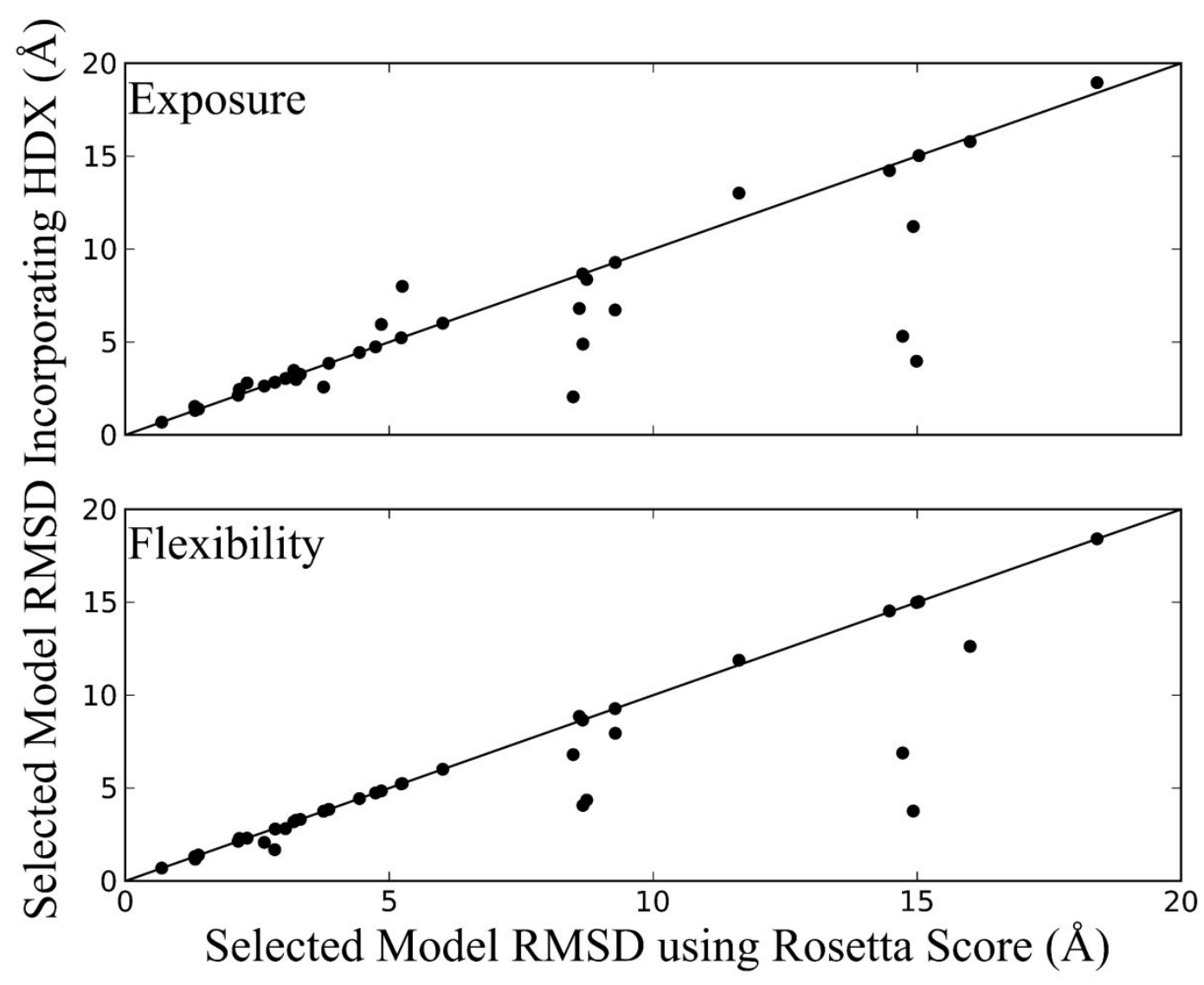


Figure S2. The average RMSD of the top 10 scoring models for the Rosetta prediction and when scoring with the HDX Score. Markers below the $y=x$ line indicate a protein with an improvement in selected model RMSD, with those above worsening.

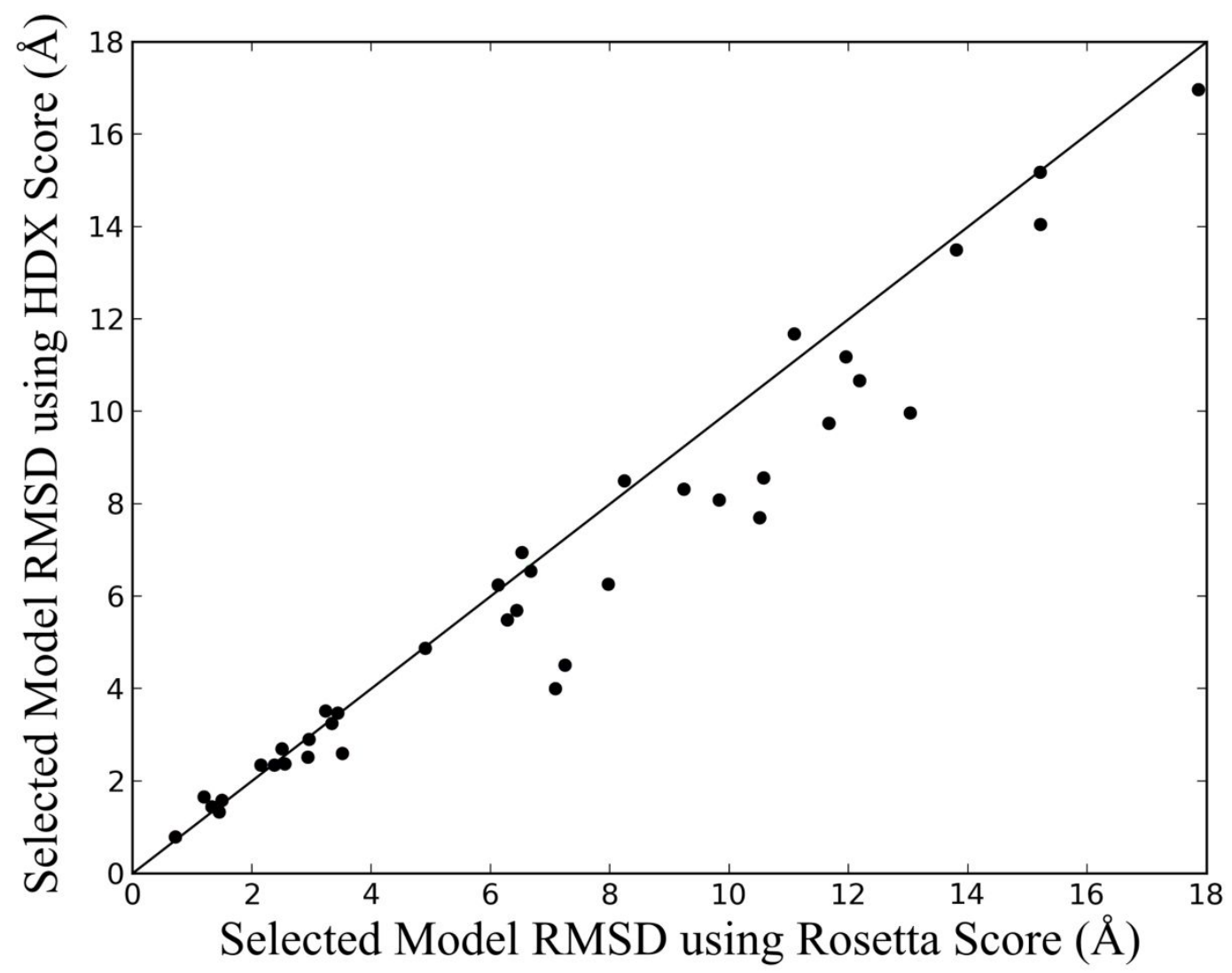


Figure S3. RMSD distribution of top 10 scoring models for all proteins in the benchmark set using Rosetta score (gray) and the combined-term HDX score (red).

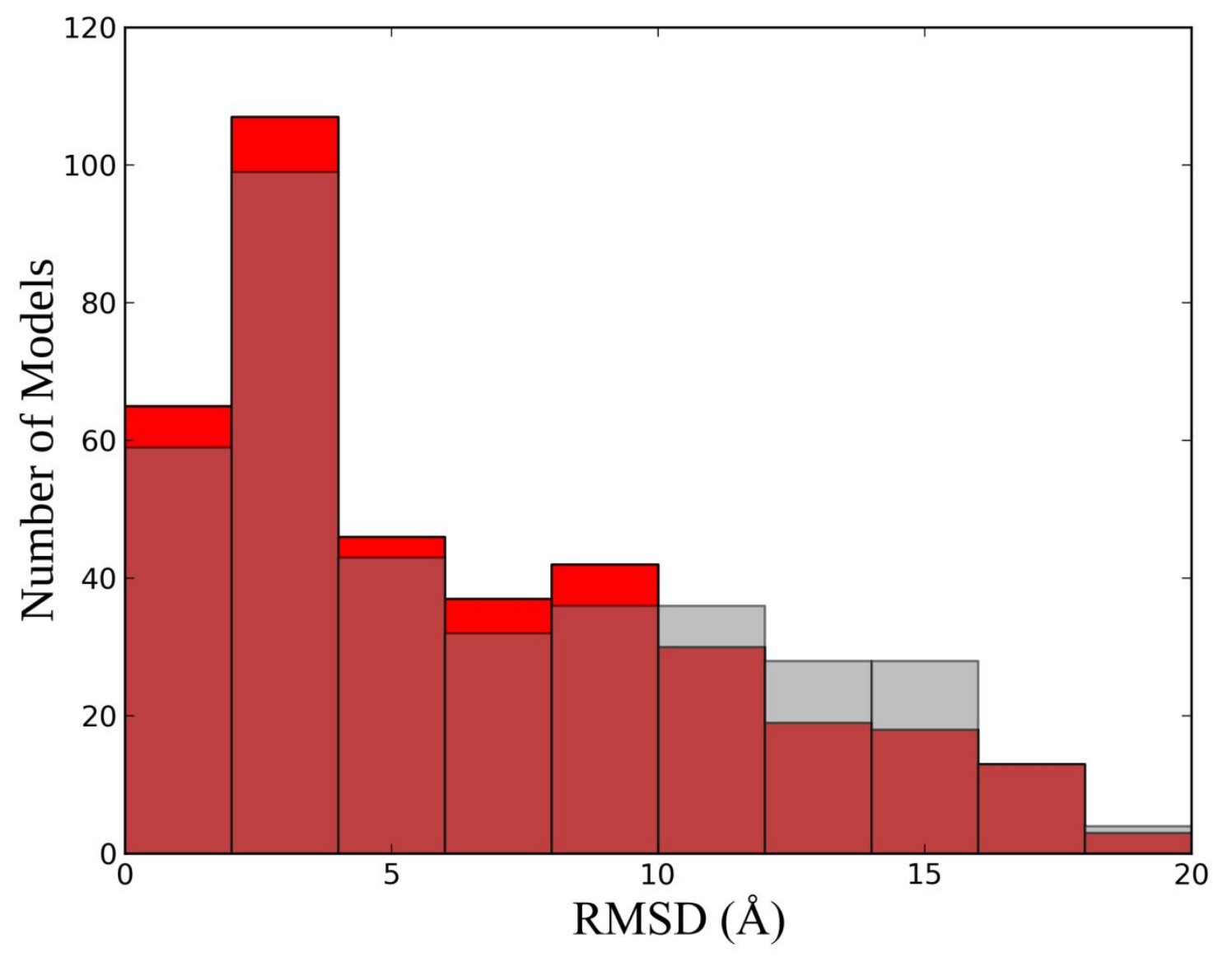


Figure S4. Rosetta (left) and HDX (right) Score vs RMSD plot of the two proteins used for the independent verification test. The lowest scoring model is marked by a black star.
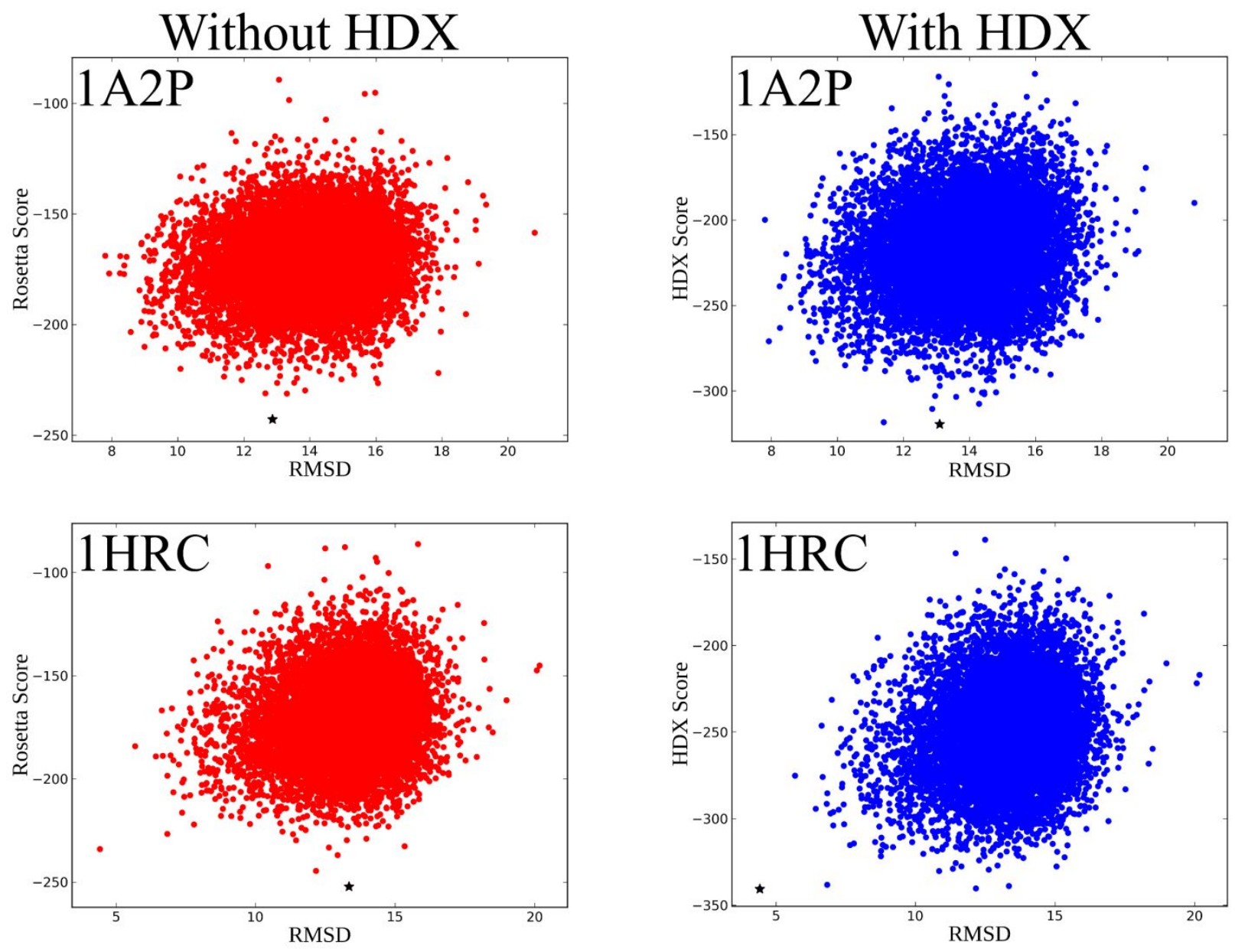